\title{
Use of LSPR Spectroscopy Biosensing for In Situ Identification of Arsenic from Bioleaching of Realgar by Acidithiobacillus ferrooxidans
}

\author{
Ruixiang Xu, ${ }^{1}$ Wenbin Zhao, ${ }^{1}$ Shenghui $X u,{ }^{2}$ Lei Yan, ${ }^{3}$ Zhengrong Wu, ${ }^{1}$ Yuheng Liu, ${ }^{1}$ \\ Wantong Ma, ${ }^{1}$ and Peng Chen $\mathbb{1}^{1}$ \\ ${ }^{1}$ School of Pharmacy, Lanzhou University, No. 199 Donggang West Road, Lanzhou 730020, China \\ ${ }^{2}$ College of Earth and Environmental Sciences, Lanzhou University, Key Laboratory of Western China's Environmental Systems, \\ Ministry of Education, Lanzhou 730000, China \\ ${ }^{3}$ College of Life Science and Technology, Heilongjiang Bayi Agricultural University, Daqing 163319, China
}

Correspondence should be addressed to Peng Chen; chenpeng@lzu.edu.cn

Received 27 August 2018; Revised 26 October 2018; Accepted 4 November 2018; Published 2 December 2018

Academic Editor: Alessandro Longo

Copyright $\odot 2018$ Ruixiang Xu et al. This is an open access article distributed under the Creative Commons Attribution License, which permits unrestricted use, distribution, and reproduction in any medium, provided the original work is properly cited.

Localized surface plasmon resonance (LSPR) spectroscopy has received extensive attention as a new method in chemical and biological analysis that can be integrated with nanotechnology. As a new analytical method, LSPR possesses various advantages over the traditional bioanalysis method of enzyme-linked immunosorbent assay (ELISA), including a label-free procedure, low cost, high response speed, simple operation and structure, and ease of storage and transport. Additionally, in situ and highthroughput measurements can be achieved. This study aims to solve the problem of the lack of in situ and highly efficient monitoring methods for current realgar bioleaching processes. An LSPR chip is made to monitor the changes in arsenic content in the process of realgar bioleaching. A convenient, fast, sensitive, and high-throughput method of bioleaching process investigation based on the LSPR spectroscopic in situ monitoring technique is proposed. The LSPR chip provided a highly specific selectivity and a linear detection of arsenic content in the range of $1.0-100.0 \mu \mathrm{M}$ with detection limit (LOD) $0.898 \mu \mathrm{M}$. The developed chip was applied to the quantification of realgar bioleaching sample with satisfactory results.

\section{Introduction}

Bioleaching technology is a newly developed hydrometallurgical technology [1]. This technique is cross-fused with the preparation of medicinal realgar and uses natural acidophilic microorganisms to perform bioleaching [2]. Recent studies have confirmed that the realgar leachate obtained using Acidithiobacillus ferrooxidans (A. ferrooxidans) showed strong anticancer activity in both in vitro and in vivo experiments [3,4]. Compared to traditional preparatory methods, the bioleaching of realgar can significantly increase its solubility and bioavailability. In addition, this method is highly efficient, ecofriendly, and of low cost [5]. In the bioleaching of realgar, an important aspect is monitoring the changes in the concentration of arsenic during the process
[2]. Traditionally, the molybdenum blue spectrophotometric method is utilized to measure the arsenic content. This method is tedious, requires a specific experimental setup, and needs to be airtight. The final gaseous arsenic product of the reaction is toxic and difficult to collect, affecting both the experimental results and the experimenters. Additionally, the experimental setup does not allow high-throughput processing in the case of high amounts. Another commonly implemented method utilizes instrumental analysis by inductively coupled plasma atomic emission spectroscopy (ICP-AES). ICP-AES is safer and more sensitive, but the instrumentation is relatively expensive, and, more importantly, it is difficult to conduct in situ analysis.

As a new analysis method, localized surface plasmon resonance (LSPR) provides various advantages compared to 
the traditional bioanalysis technique enzyme-linked immunosorbent assay (ELISA) [6]. These advantages include labelfree procedures, low cost, high response speed, simple operation and structure, and ease of storage and transport [7]. In addition, in situ and high-throughput measurements can be achieved. In recent years, there have been extensive advances in synthetic nanotechnology [8]. It is possible to control and synthesize nanoparticles with specific morphologies, sizes, and properties according to the need. For precious metal nanoparticles (NPs) that are specifically utilized in LSPR analysis, the refractive index sensitivities for the dielectric environment of various types of NPs are in the following order: nanostars > nanoprisms > bipyramids > nanorods $>$ nanocubes nanospheres [9]. The LSPR sensitivity of nonspheroidal particles cannot be described analytically, but it has been shown experimentally and in electrodynamics simulations that particle shape plays a large role in determining the sensitivity [10]. In particular, particles with sharp tips produce much higher refractive index sensitivities than would be predicted from their aspect ratios alone. Over the past decade, a myriad of new nanoparticle shapes with ever-increasing refractive index sensitivities have been developed [11]. In this study, we selected the triangular Ag@Au core-shell NP as the nanostructure on which the LSPR analysis is based. Ag@Au core-shell NPs possess the following advantages: (I) for the Ag@Au core-shell NP we synthesized, the refractive index sensitivity to the media environment could reach $570 \mathrm{~nm} /$ RIU. This sensitivity is significantly greater than that of the previously reported triangular silver NPs and spherical gold and silver NPs [12]. (II) Conceptually, Ag NPs, due to the stronger and narrower plasmon resonance peak, could be widely adapted for use in chemical sensors, biosensors, and imaging and catalytic applications. However, the structure of silver NPs can change under conditions such as in the presence of salt solutions, halogens, oxidants, heat, and ultraviolet light [13-16].

This property has greatly limited the practical application of silver NPs in various areas. Nevertheless, when a triangular silver NP is coated with an even, thin layer of gold, the stability can be greatly increased, thus increasing the value of silver NPs in practical applications [17]. Gold (III) NPs are easy to modify and exhibit good biocompatibility [18]. Moreover, coating silver NPs with a layer of gold not only effectively protects the silver NPs from environmental influences but also effectively improves the physical and chemical properties of the silver NPs [19]. Therefore, this study provides a new solution to the difficulty of monitoring the change in arsenic content in the process of bioleaching realgar by producing an LSPR chip to investigate the bioleaching process via a convenient, rapid, sensitive, and highthroughput LSPR spectroscopic in situ monitoring technique.

In summary, this work is focused on the following tasks: (1) prepare triangular Ag@Au core-shell NPs, based on which a chip is produced using Ag@Au triangular NPs, and perform further characterization; (2) investigate realgar bioleaching by $A$. ferrooxidans and implement the prepared LSPR chip to monitor changes in arsenic content in the bioleaching process.

\section{Materials and Methods}

2.1. Realgar Bioleaching System. The strain A. ferrooxidans BY-3 (CCTCC-M203071) was obtained from the discarded copper mine in Baiyin City, Gansu Province, China, and purified by solid medium streak-plate separation. This strain was selected based on its growth and physical and chemical properties. The strain was entered into GenBank after 16S rRNA nucleic acid sequencing (DQ676505) [20]. The culture medium for strain A. ferrooxidans BY-3 (CCTCC-M203071) was 9K culture medium containing $44.69 \mathrm{~g} / \mathrm{L}$ ferrous sulfate [21]. A. ferrooxidans is used to leach out the arsenic within realgar in water solutions. Using a $10 \%$ inoculum volume, strain A. ferrooxidans BY-3 was inoculated into the culture medium containing $5 \mathrm{~g} / \mathrm{L}$ of realgar powder. The $\mathrm{pH}$ was adjusted to 1.7 , the agitation speed was $150 \mathrm{r} / \mathrm{min}$, and the bacteria were cultured in a shaker at a constant temperature of $30^{\circ} \mathrm{C}$ for $100 \mathrm{~h}$ until the logarithmic growth phase.

2.2. Preparation of Triangular Ag@Au Core-Shell Nanoparticles. Fixed amounts of $\mathrm{AgNO}_{3}$ and sodium citrate solutions were added to $\mathrm{H}_{2} \mathrm{O}_{2}$. Then, triangular silver NPs were synthesized by adding a fixed amount of freshly prepared cold $\mathrm{NaBH}_{4}$ solution while stirring the mixture. The resulting triangular silver NP solution was homogeneously mixed with fixed amounts of $3 \mathrm{~mL}$ PVP (5\%), $450 \mu \mathrm{l}$ diethylamine, and $1.2 \mathrm{~mL}$ ascorbic acid solutions $(0.05 \mathrm{M})$. The mixture, while stirring for $1 \mathrm{~h}$, reacted with the $0.6 \mathrm{~mL}$ $\mathrm{HAuCl}_{4}$ growth solution, $1.2 \mathrm{~mL}$ PVP (5\%), $240 \mu \mathrm{KI}$, and $9 \mathrm{~mL} \mathrm{H}_{2} \mathrm{O}$ and produced Ag@Au core-shell triangular NPs $(\sim 0.4 \mathrm{nM})$ [22].

\subsection{Preparation of Chip Based on Ag@Au Triangular Nanoparticles}

2.3.1. Pretreatment of Glass Substrate. Standard plain microscope glass slides were cut into small pieces. The glass pieces were cleaned and then sonicated in piranha acid solution and ammonia solution for surface hydroxylation. The glass pieces were then rinsed with a large volume of pure water, blow-dried with argon, and soaked in APTES ((3aminopropyl)triethoxysilane) solution for surface amination. The surface-aminated glasses were further sonicated in methanol, rinsed with a large volume of pure water, blowdried with argon, and oven-dried.

2.3.2. Assembly of Ag@Au Core-Shell Nanoparticles on Glass Slide Surface. Pretreated glass slides were placed on a watch glass. A certain concentration of Ag@Au NP solution was poured into the watch glass to a certain level above the slide surface. After a period for assembly, the glass slides were removed and washed with a large volume of ultrapure water, blow-dried with argon, and analyzed by UV-Vis extinction spectroscopy. 

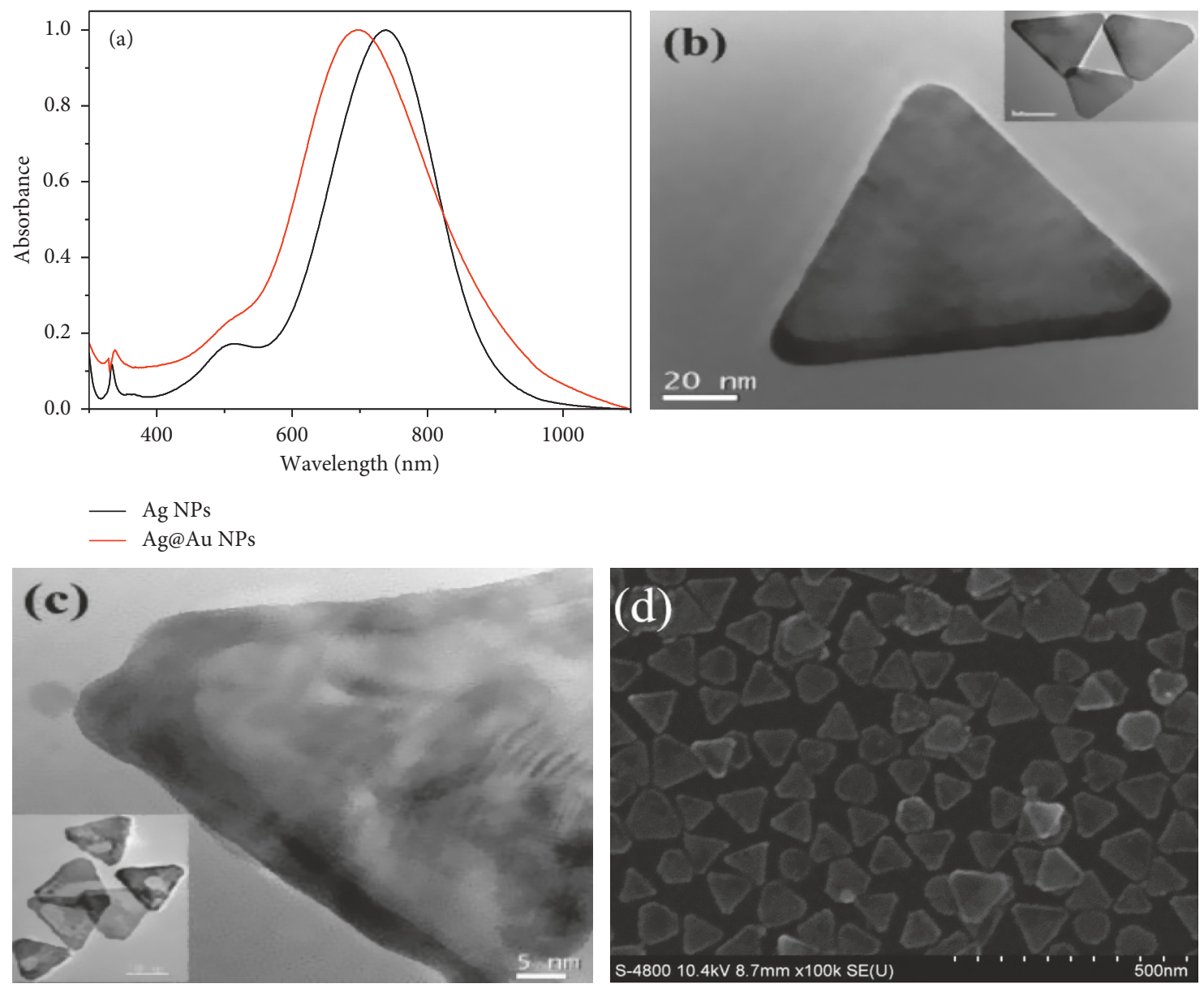

Figure 1: (a) UV-Vis extinction spectra of prepared triangular silver NPs (black line) and Ag@Au core-shell NPs (red line). (b) and (c) TEM images of triangular silver NPs and Ag@Au core-shell NPs. (d) A scanning electron microscopy image of Ag@Au core-shell NPs.

\section{Results and Discussion}

\subsection{Preparation and Property Tests of Ag@Au Triangular Nanoparticle Chip}

3.1.1. Preparation of Triangular Ag@Au Core-Shell Nanoparticles. Figure 1 displays the UV-Vis extinction spectra of the prepared triangular silver NPs (Figure 1(a), black line) and the Ag@Au core-shell NPs (Figure 1(a), red line). Figures 1(b) and 1(c) show the transmission electron microscopy (TEM) images of the triangular silver NPs and the Ag@Au core-shell NPs. Figure 1(d) shows the scanning electron microscopy image of the Ag@Au coreshell NPs.

3.1.2. Evaluation of the Stability of Ag@Au Core-Shell Nanoparticles. As seen in Figure 2, the stability of the prepared Ag@Au core-shell triangular NPs was fairly good. The NPs were stably present in buffer and salt solutions. Note that the position of the maximum LSPR peaks $\left(\lambda_{\max }\right)$ was almost unchanged, but the intensity was decreased. The main cause was the trace coagulation of NPs in high concentrations of PBS buffer or salt solution and a certain amount of loss of NPs during each measurement. In our study, LSPR measurement is mainly based on the position change of the maximum LSPR peaks, and thus, the change in intensity does not influence the measurement results.

3.1.3. Evaluation of the Sensitivity of the Ag@Au Core-Shell Nanoparticles. As illustrated in Figure 3, the detection sensitivity of this type of core-shell NP in solution was high. From a linear regression analysis of the relationship between the LSPR $\lambda_{\max }$ position of the core-shell NPs and the solution refractive index $(n)$, the sensitivity could reach as high as $570 \mathrm{~nm} / \mathrm{RIU}$.

3.1.4. Preparation of Ag@Au Triangular Nanoparticle Chip. The core-shell NPs to be assembled onto glass slides still showed localized surface plasmon resonance and could thus result in LSPR peaks. Meanwhile, due to the difference in refractive indexes of the air, glass, and water, a blue shift of LSPR peaks, as illustrated in Figure 4(a), was observed for glass slides loaded with NPs. The peak shape was similar to the peak shape of the monodispersed Ag@Au core-shell NPs in aqueous solution. On the other hand, upon observing the glass slides after assembly, the color of the slides was 


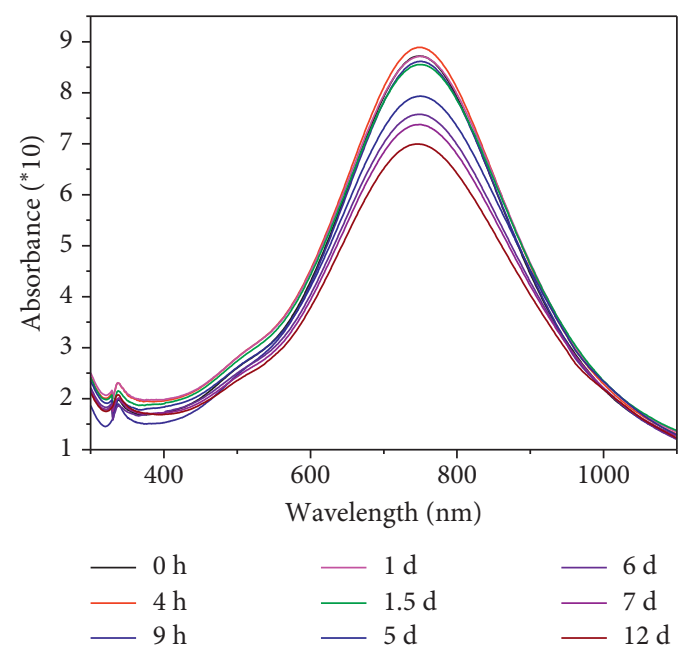

(a)

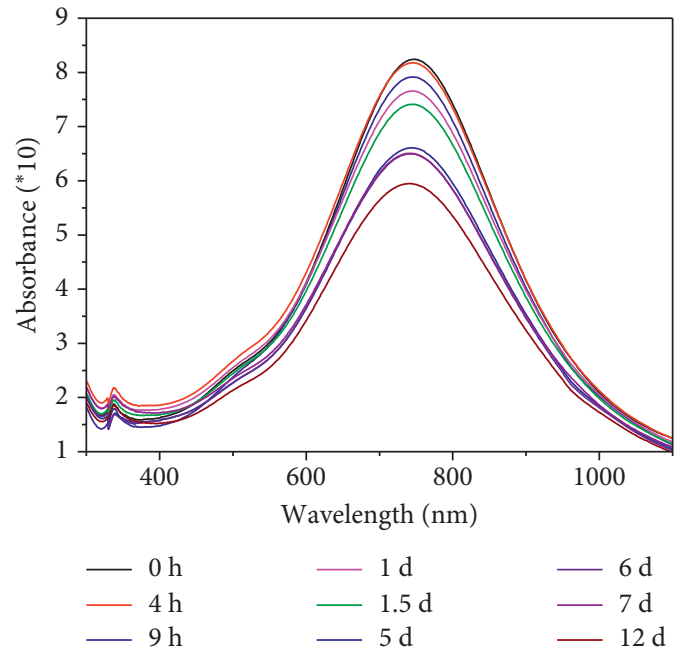

(b)

Figure 2: UV-Vis extinction spectra of Ag@Au core-shell NPs stored for a period of time in pure water (a) and PBS buffer (10 mM, pH=7.4) (b).

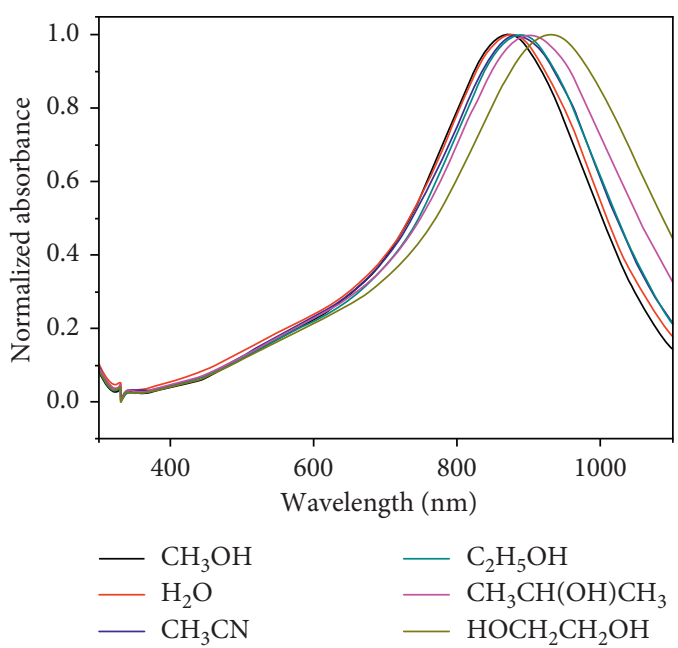

(a)

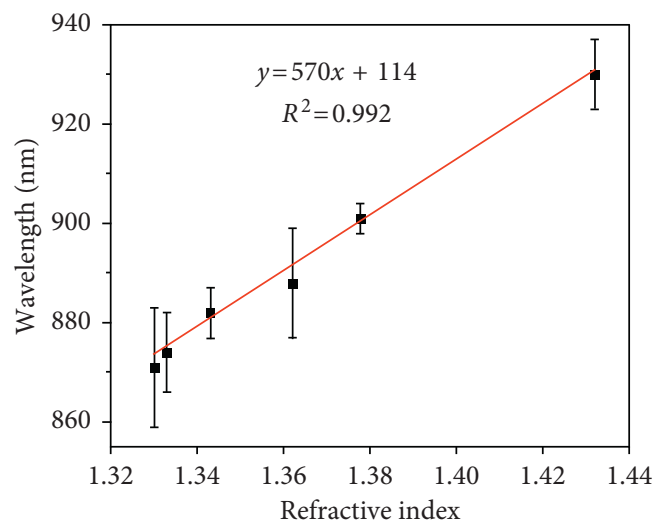

(b)

Figure 3: (a) UV-IR extinction spectra of Ag@Au NPs in 6 different solvents: $\mathrm{CH}_{3} \mathrm{OH}(n=1.3301), \mathrm{H}_{2} \mathrm{O}(n=1.333), \mathrm{CH}_{3} \mathrm{CN}(n=1.343)$, $\mathrm{C}_{2} \mathrm{H}_{5} \mathrm{OH}(n=1.362), \mathrm{CH}_{3}(\mathrm{OH}) \mathrm{CH}_{3} \mathrm{CH}(n=1.3776)$, and $\mathrm{HOCH}_{2} \mathrm{CH}_{2} \mathrm{OH}(n=1.4318)$. (b) Relationship between the LSPR $\lambda_{\text {max }}$ shift of Ag@ $\mathrm{Au}$ core-shell NPs and the refractive index $(n)$ of the solvent.

similar to the color of the monodispersed NPs; both were blue, but the glass slide was of lighter color. These two observations suggest that when NPs assembled on the glass surface, they were fixed onto the slides in a nonaggregated state. As shown in Figure 4(b), the full-width half-maximum of the LSPR peak was narrowest for slides soaked for $2 \mathrm{~h}$, so the soaking time was set at $2 \mathrm{~h}$ for the subsequent experiments.

3.2. Application of LSPR Chip in Monitoring the Change in Arsenic Content in the Bioleaching Process. Glutathione (GSH) and dithiothreitol (DTT) were treated as recognition units and modified onto the Ag@Au core-shell NP chip through the Au-S chain reaction. Acidophilic bacteria, such as $A$. ferrooxidans, can increase the solubility of realgar, and the main reactions are as follows:

$$
\begin{aligned}
\mathrm{As}_{2} \mathrm{~S}_{2}+14 \mathrm{H}_{2} \mathrm{O} \stackrel{\text { A.ferrooxidans }}{\longrightarrow} & 2 \mathrm{H}_{3} \mathrm{AsO}_{3} \\
& +2 \mathrm{HSO}_{4}{ }^{-}+20 \mathrm{H}^{+}+18 \mathrm{e}^{-}
\end{aligned}
$$

$$
\mathrm{H}_{3} \mathrm{AsO}_{3}+\mathrm{H}_{2} \mathrm{O} \stackrel{\text { A.ferrooxidans }}{\longrightarrow} \mathrm{H}_{3} \mathrm{AsO}_{4}+2 \mathrm{H}^{+}+2 \mathrm{e}^{-}
$$

The sulfhydryl group of GSH or DTT can react with arsenate and reduce $\mathrm{As}(\mathrm{V})$ to $\mathrm{As}(\mathrm{III})$. As(III) can form the As- $(\mathrm{SG})_{3}$ complex with GSH in solution, while $\mathrm{As}(\mathrm{V})$ can 


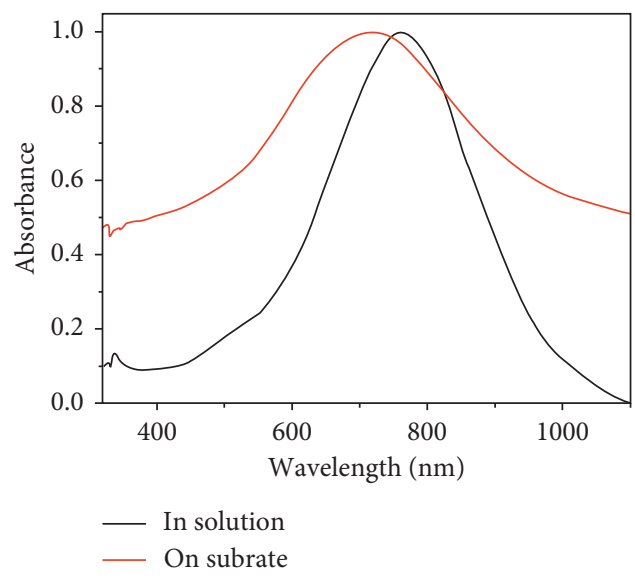

(a)

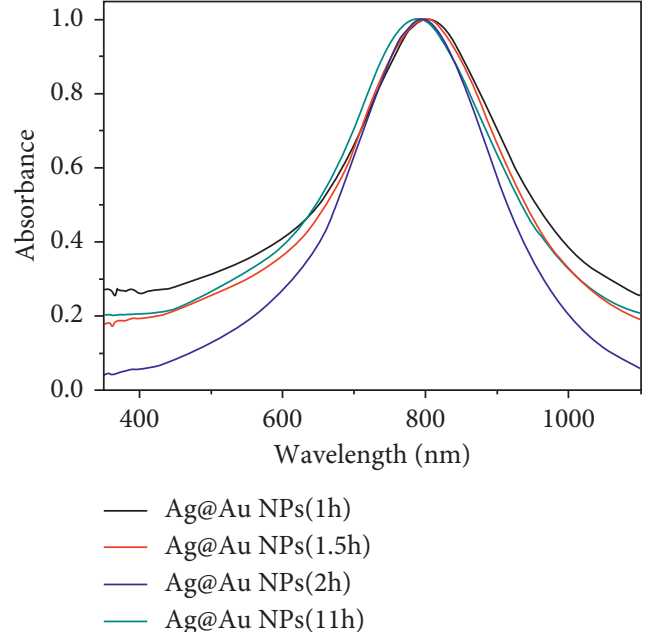

(b)

FIgURE 4: (a) UV-IR extinction spectra of Ag@Au core-shell NPs in aqueous solution and assembled on glass slides. (b) LSPR peaks, before and after normalization, of glass slides soaked in Ag@Au core-shell NP solution for different periods of time.

oxidize GSH to form oxidized GSH, GS-SG. The main mechanisms are as follows:

$$
\begin{aligned}
\mathrm{H}_{2} \mathrm{AsO}_{4}^{-}+5 \mathrm{GSH}+\mathrm{H}^{+} \longrightarrow \mathrm{As} & -(\mathrm{SG})_{3}(\mathrm{III}) \\
& +\mathrm{GS}-\mathrm{SG}+4 \mathrm{H}_{2} \mathrm{O} \\
\mathrm{HAsO}_{4}{ }^{2-}+5 \mathrm{GSH}+2 \mathrm{H}^{+} \longrightarrow \mathrm{As} & -(\mathrm{SG})_{3}(\mathrm{III}) \\
& +\mathrm{GS}-\mathrm{SG}+4 \mathrm{H}_{2} \mathrm{O}
\end{aligned}
$$

As recognition units, GSH and DTT can be modified onto the Ag@Au core-shell NP chip by the Au-S chain reaction. When the GSH or DTT on glass slides reacts with arsenate to form the As- $(\mathrm{SG})_{3}$ complex and GS-SG, the refractive index of the LSPR chip surface is altered, resulting in changes to the LSPR peak. The goal of analysis can then be achieved by UV-IR absorbance spectroscopic characterization. The analysis mechanism is illustrated in Figure 5. The LSPR chip was used through UV-Vis-IR extinction spectroscopy analysis to perform a preliminary analysis of arsenate solution (Figure 6). Application of the LSPR chip method showed the linear range of arsenate in the bioleaching process from $1.0 \mu \mathrm{M}$ to $100.0 \mu \mathrm{M}$, and a detection limit of $0.898 \mu \mathrm{M}$ was obtained.

\section{Conclusion}

Triangular Ag@Au core-shell NPs were successfully prepared, and Ag@Au triangular NP chips were prepared and characterized. The process of realgar bioleaching by $A$. ferrooxidans was studied. The prepared LSPR chip was applied to monitor the change in arsenic content in the bioleaching process. Additionally, it was found in the experimental process that the realgar bioleaching process by $A$. ferrooxidans could accelerate the oxidation of the ferrous ion. The resulting arsenic ion can be detected by a type of new chips, which provides an alternative method of

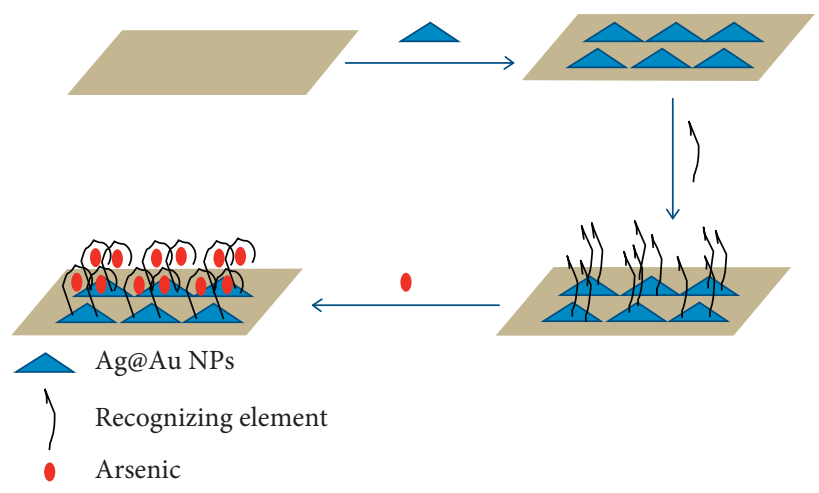

FIGURE 5: Mechanism flow chart of the preparation of the Ag@Au core-shell NP chip and its application in analysis.

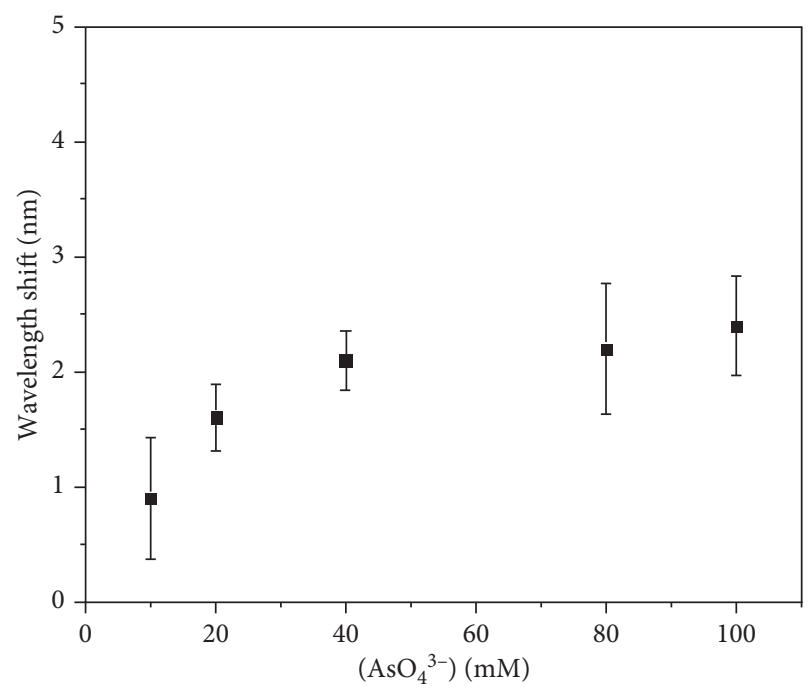

FIGURE 6: Preliminary analysis of arsenate by the LSPR chip. 
monitoring by observing changes in arsenic content in the bioleaching process.

\section{Data Availability}

The data used to support the findings of this study are available from the corresponding author upon request.

\section{Conflicts of Interest}

The authors declare that there are no conflicts of interest.

\section{Authors' Contributions}

Both authors approve the final version of the manuscript.

\section{Acknowledgments}

This work was supported by the Technology Program of Gansu Province (Grant Nos. 1604FKCA110 and 1606RJZA087), the Fundamental Research Funds for the Central Universities of China (Grant No. lzujbky-2017-197), the Project of Lanzhou City for Innovative and Entrepreneurial Talents (Grant No. 2017-RC-73), and the Science and Technology Project of Lanzhou City (Grant Nos. 2015-3-93, 2016-3-75, 2017-4-122, and 2018-4-59).

\section{References}

[1] J. H. Zhang, X. Zhang, Y. Q. Ni, X. J. Yang, and H. Y. Li, "Bioleaching of arsenic from medicinal realgar by pure and mixed cultures," Process Biochemistry, vol. 42, no. 9, pp. 1265-1271, 2007.

[2] P. Chen, L. Yan, F. F. Leng et al., "Bioleaching of realgar by Acidithiobacillus ferrooxidans using ferrous iron and elemental sulfur as the sole and mixed energy sources," Bioresource Technology, vol. 102, no. 3, pp. 3260-3267, 2011.

[3] S. Peng, C. Peng, W. Dong et al., "Realgar transforming solution displays anticancer potential against human hepatocellular carcinoma HepG2 cells by inducing ROS," International Journal of Oncology, vol. 50, no. 2, pp. 660-670, 2017.

[4] X. Wang, X. Zhang, Z. Xu, Z. Wang, X. Yue, and H. Li, "Reversal effect of arsenic sensitivity in human leukemia cell line K562 and K562/ADM using Realgar Transforming Solution," Biological and Pharmaceutical Bulletin, vol. 36, no. 4, pp. 641-648, 2013.

[5] P. Chen, L. Yan, X. Yue, and H. Li, "Optimal parameters for bioleaching of realgar using Acidithiobacillus ferrooxidans under different growth conditions and mathematical analysis," Biocatal Biotransformation, vol. 31, no. 1, pp. 33-41, 2013.

[6] K. M. Mayer and J. H. Hafner, "Localized surface plasmon resonance sensors," Chemical Reviews, vol. 111, no. 6, pp. 3828-3857, 2011.

[7] E. Petryayeva and U. J. Krull, "Localized surface plasmon resonance: nanostructures, bioassays and biosensing-a review," Analytica Chimica Acta, vol. 706, no. 1, pp. 8-24, 2011.

[8] S. Szunerits and R. Boukherroub, "Sensing using localised surface plasmon resonance sensors," Chemical Communications, vol. 48, no. 72, pp. 8999-9010, 2012.

[9] L. B. Sagle, L. K. Ruvuna, J. A. Ruemmele, and R. P. Van Duyne, "Advances in localized surface plasmon resonance spectroscopy biosensing," Nanomedicine, vol. 6, no. 8, pp. 1447-1462, 2011.

[10] K. M. Mayer and J. H. Hafner, "Localized surface plasmon resonance sensors," Chemical Reviews, vol. 111, no. 6, pp. 3828-3857, 2011.

[11] T. K. Sau, A. L. Rogach, F. Jäckel, T. A. Klar, and J. Feldmann, "Properties and applications of colloidal nonspherical noble metal nanoparticles," Advanced Materials, vol. 22, no. 16, pp. 1805-1825, 2010.

[12] M. Rycenga, C. M. Cobley, J. Zeng et al., "Controlling the synthesis and assembly of silver nanostructures for plasmonic applications," Chemical Reviews, vol. 111, no. 6, pp. 3669$3712,2011$.

[13] K. A. Homan, M. Souza, R. Truby et al., "Silver nanoplate contrast agents for in vivo molecular photoacoustic imaging," ACS Nano, vol. 6, no. 1, pp. 641-650, 2012.

[14] M. Kim, Y. W. Lee, D. Kim et al., "Reshaping nanocrystals for tunable plasmonic substrates," ACS Applied Materials and Interfaces, vol. 4, no. 9, pp. 5038-5043, 2012.

[15] Q. Zhang, J. Ge, T. Pham et al., "Reconstruction of silver nanoplates by UV irradiation: tailored optical properties and enhanced stability," Angewandte Chemie International Edition, vol. 48, no. 19, pp. 3516-3519, 2009.

[16] Q. Zhang, N. Li, J. Goebl, Z. Lu, and Y. Yin, "A systematic study of the synthesis of silver nanoplates: is citrate a "magic" reagent?," Journal of the American Chemical Society, vol. 133, no. 46, pp. 18931-18939, 2011.

[17] W. P. Hall, S. N. Ngatia, and R. P. Van Duyne, "LSPR biosensor signal enhancement using nanoparticle-antibody conjugates," Journal of Physical Chemistry C, vol. 115, no. 5, pp. 1410-1414, 2011.

[18] Y. Cui, B. Ren, J. L. Yao, R. A. Gu, and Z. Q. Tian, "Synthesis of AgcoreAushell bimetallic nanoparticles for immunoassay based on surface-enhanced Raman spectroscopy," Journal of Physical Chemistry B, vol. 110, no. 9, pp. 4002-4006, 2006.

[19] H. He, X. Xu, H. Wu, and Y. Jin, "Enzymatic plasmonic engineering of $\mathrm{Ag} / \mathrm{Au}$ bimetallic nanoshells and their use for sensitive optical glucose sensing," Advanced Materials, vol. 24, no. 13, pp. 1736-1740, 2012.

[20] Y. Ni, D. Wan, and K. He, "16S rDNA and 16S-23S internal transcribed spacer sequence analyses reveal inter- and intraspecific Acidithiobacillus phylogeny," Microbiology, vol. 154 , no. 8, pp. 2397-2407, 2008.

[21] P. Chen, L. Yan, Q. Wang, Y. Li, and H. Li, "Surface alteration of realgar $\left(\mathrm{As}_{4} \mathrm{~S}_{4}\right)$ by Acidithiobacillus ferrooxidans," International Microbiology, vol. 15, no. 1, pp. 9-15, 2012.

[22] Q. Zhang, Y. Hu, S. Guo, J. Goebl, and Y. Yin, "Seeded growth of uniform Ag nanoplates with high aspect ratio and widely tunable surface plasmon bands," Nano Letters, vol. 10, no. 12, pp. 5037-5042, 2010. 

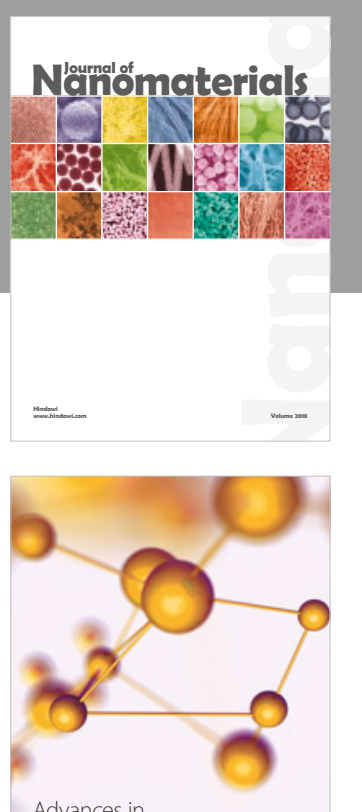

Physical Chemistry
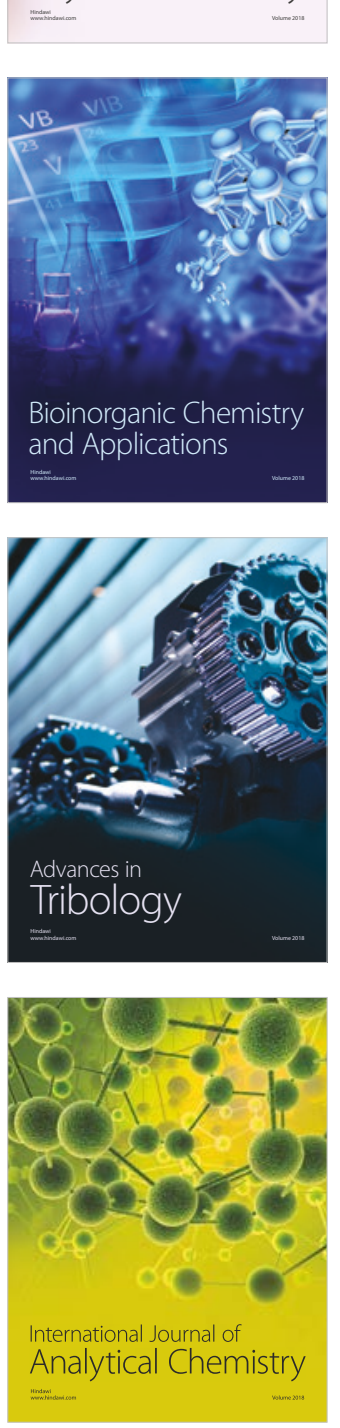

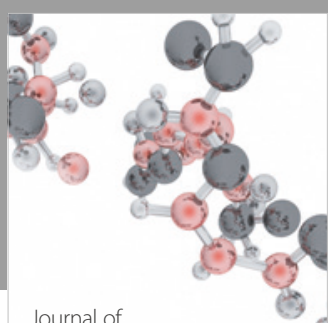

Analytical Methods

in Chemistry

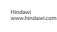

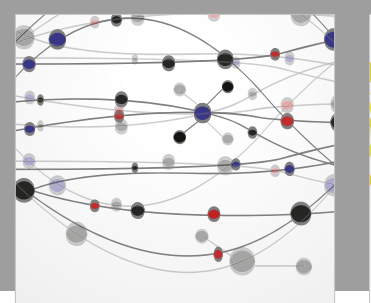

The Scientific World Journal

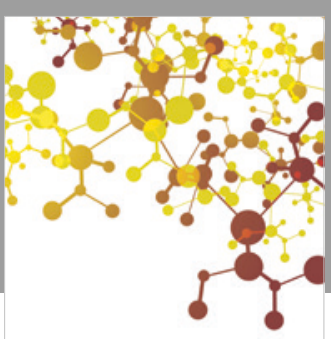

Journal of

Applied Chemistry
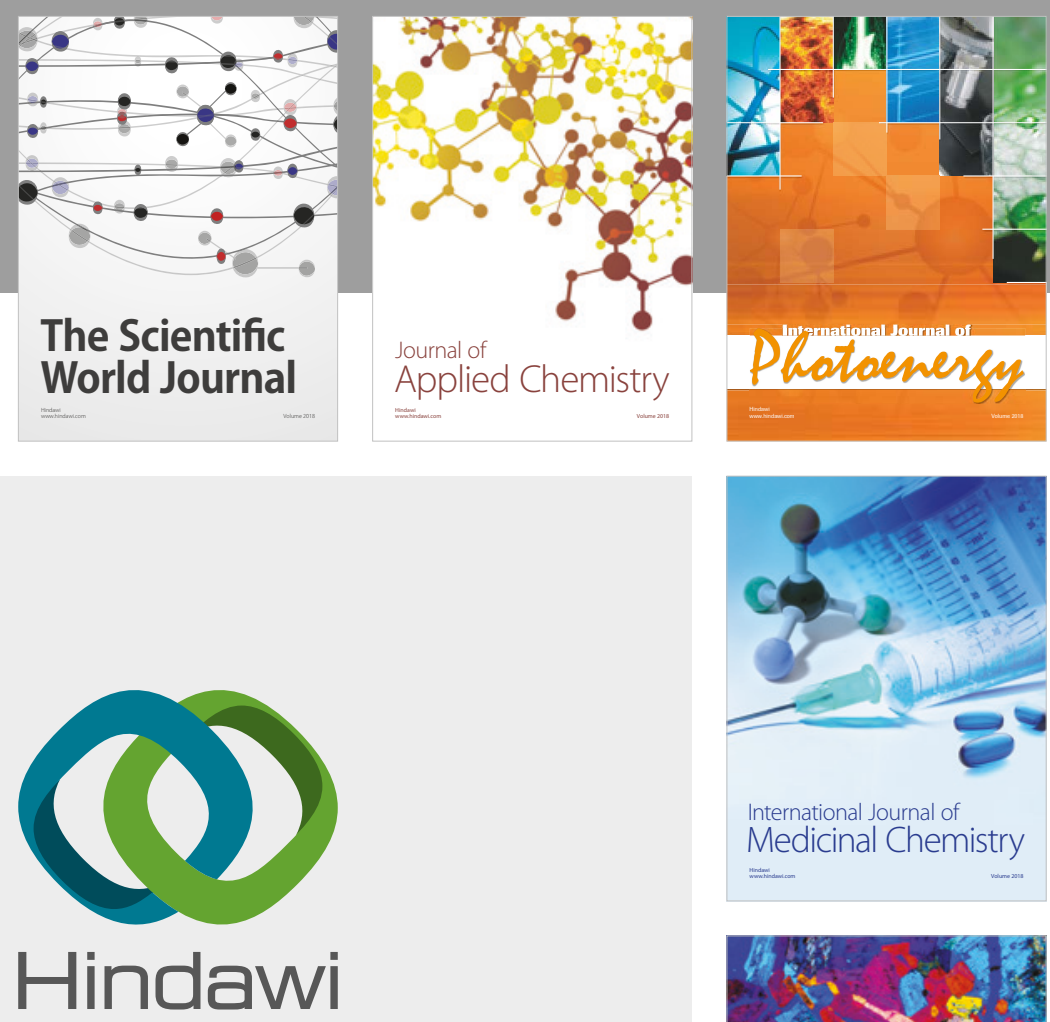

Submit your manuscripts at

www.hindawi.com
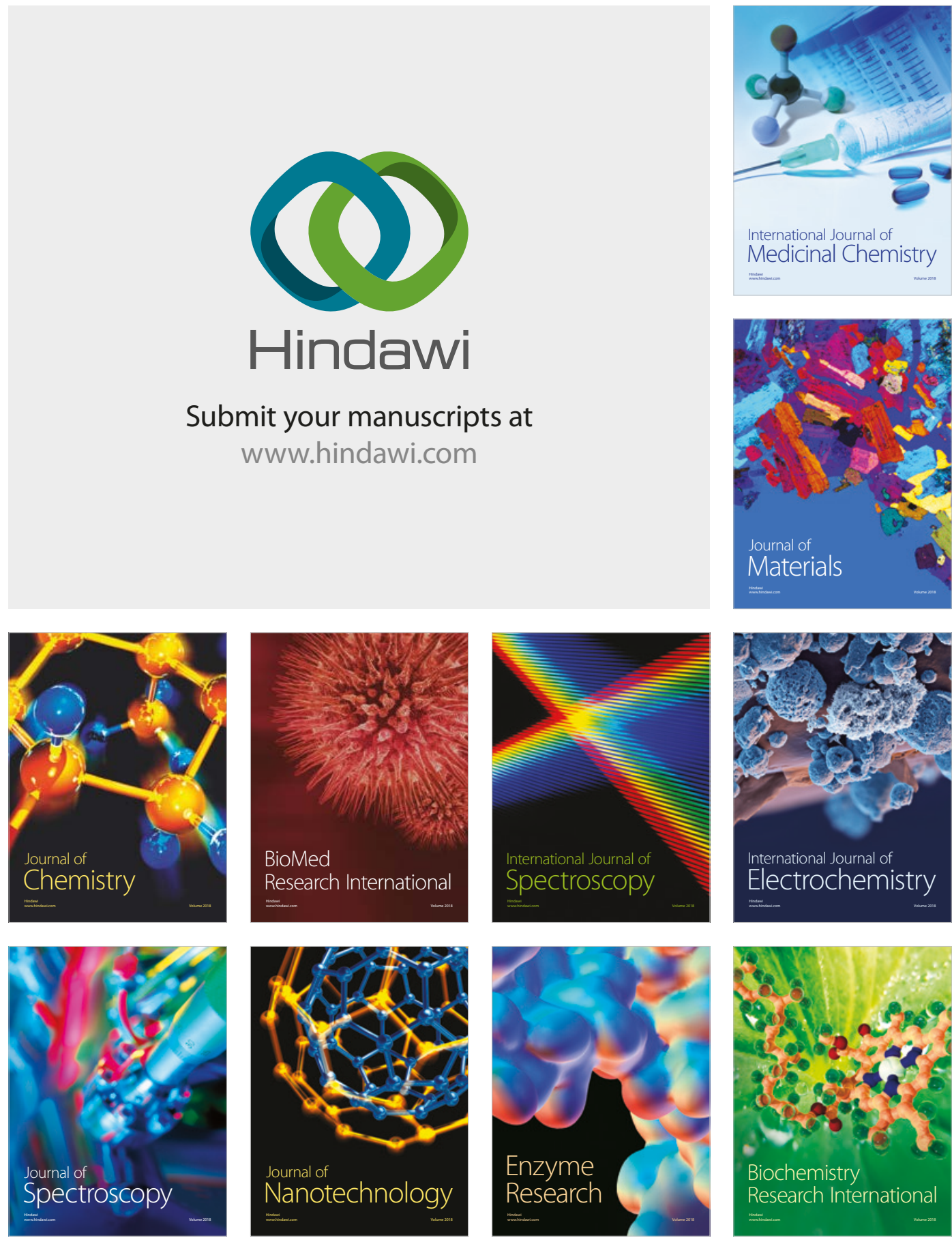
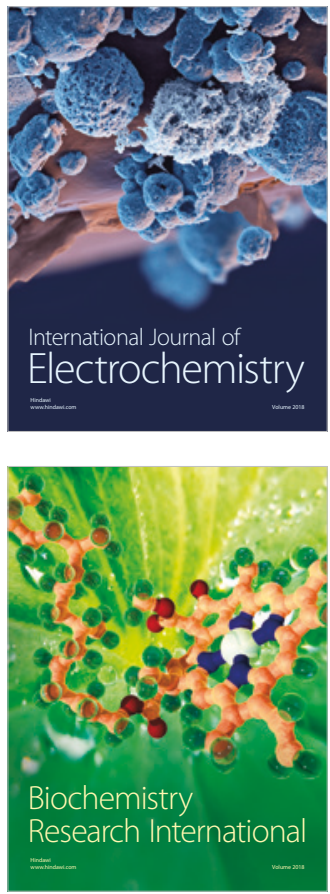